\title{
BREVE ANÁLISE DA EVOLUÇAO E STATUS QUO DAS UNIDADES DE CONSERVAÇÃO NO CERRADO BRASILEIRO
}

\author{
Carlos Vinícius Gonçalves Ribeiro \\ Universidade Federal de Lavras (UFLA), Departamento de Ciências Florestal \\ Pós-Graduando em Engenharia Florestal, Lavras, MG, Brasil \\ carlos.engenhariaflorestal@gmail.com \\ Luís Antônio Coimbra Borges \\ Universidade Federal de Lavras(UFLA), Departamento de Ciências Florestal, Lavras, MG, Brasil \\ luis.borges@ufla.br
}

\begin{abstract}
RESUMO
Os altos níveis de exploração dos recursos naturais resultaram em uma elevada degradação ambiental, provocando impactos negativos na biodiversidade dos ecossistemas naturais. Uma das ferramentas para minimizar os impactos na natureza é a criação de Unidades de Conservação (UCs). Buscando compreender a situação das UCs no Cerrado brasileiro, este estudo tem como objetivo identificar a evolução das UCs no bioma ao longo de 70 anos. Os dados utilizados na pesquisa foram coletados no banco de cadastro de UCs do Ministério do Meio Ambiente. Observou-se que as primeiras UCs foram criadas em 1949 e o período de maior crescimento de UCs ocorreu entre 1990 e 2009. Constatou-se que apenas $8,72 \%$ do território do Cerrado encontra-se atualmente protegido. A maior parte das UCs estão enquadradas no grupo de Uso Sustentável, pertencem a esfera administrativa federal e não possuem plano de manejo. É necessário um melhor planejamento na criação e gestão dessas áreas, assim como, deve se adotar critérios para definição do tamanho e alocação das unidades na paisagem, visando garantir a maior conservação da biodiversidade do bioma Cerrado.
\end{abstract}

Palavras-chave: Áreas Protegidas. Cerrado. Gestão ambiental. Planejamento territorial.

\section{BRIEF ANALYSIS OF THE EVOLUTION AND STATUS QUO OF CONSERVATION UNITS IN THE BRAZILIAN CERRADO}

\begin{abstract}
The high levels of exploitation of natural resources have resulted in high environmental degradation, causing negative impacts on the biodiversity of natural ecosystems. One of the tools to minimize impacts on nature is the creation of Conservation Units (CUs). Seeking to understand the situation of CUs in the Brazilian Cerrado, this study aims to identify the evolution of CUs in the biome over 70 years. The data used in the research were collected in the CUs database of the Ministry of the Environment. It was observed that the first CUs were created in 1949 and the period of greatest growth of CUs occurred between 1990 and 2009 It was found that only $8.72 \%$ of the Cerrado's territory is currently protected. Most CUs are part of the Sustainable Use group, belong to the federal administrative sphere and do not have a management plan. Better planning is required in the creation and management of these areas, as well as criteria for defining the size and allocation of units in the landscape, in order to ensure greater conservation of biodiversity in the Cerrado biome.
\end{abstract}

Keywords: Protected Areas. Cerrado. Environmental Management. Territorial planning.

\section{INTRODUÇÃO}

O Cerrado é o segundo maior bioma do Brasil ocupando 24\% do território nacional (COUTINHO, 2016). Essa região abriga $1,5 \%$ do total de plantas vasculares do mundo e cerca de $80 \%$ dessas são endêmicas (ARRUDA et al., 2018; VIEIRA et al., 2018). No entanto, é um dos domínios florísticos mais ameaçados do mundo, visto que, aproximadamente, $50 \%$ de suas áreas foram convertidas em terras urbanas, de pastagens e cultivos agrícolas, restando 19,8\% de vegetação original (STRASSBURG et al., 2017; SANO et al., 2019). A porcentagem de áreas alteradas ou degradadas tendem a crescer nas próximas décadas, dada a expansão do agronegócio e a estagnação das ações de políticas ambientais. Esse cenário desencadeia uma série de consequências a natureza, como a fragmentação de

$\begin{array}{lllll}\text { Caminhos de Geografia } & \text { Uberlândia-MG } & \text { v. 22, n. } 83 & \text { out./2021 } & \text { p. 133-143 }\end{array}$


ecossistemas, perda de biodiversidade de fauna e flora, invasão de espécies exóticas, degradação do solo e contaminação das águas (AZEVEDO-SANTOS et al., 2018; OLIVEIRA et al., 2018).

A fim de reverter os impactos decorrentes da conversão de terras de vegetação nativa em outros usos, um conjunto de áreas protegidas foram gradualmente sendo estabelecidos (MARQUES; PERES, 2015). A lei no 9.985/2000 criou o Sistema Nacional de Unidades de Conservação (SNUC), estabelecendo normas para a criação, execução e gestão das Unidades de Conservação (UCs) (BRASIL, 2000). O SNUC separa as áreas protegidas em dois grupos: Proteção Integral (PI) e Uso Sustentável (US). O primeiro proíbe o uso e a exploração direta de recursos naturais e inclui cinco categorias: Estação Ecológica, Reserva Biológica, Parque, Monumento Natural e Refúgio de Vida Silvestre. E o segundo inclui sete categorias onde o uso direto dos recursos naturais são permitidos sob regulamentação: Área de Proteção Ambiental, Área de Relevante Interesse Ecológico, Floresta, Reserva Extrativista, Reserva de Fauna, Reserva de Desenvolvimento Sustentável e Reserva Particular do Patrimônio Natural. A criação de diferentes categorias está na necessidade de atingir diversos objetivos, pois cada categoria apresenta suas particularidades, devendo possuir manejos distintos visando atingir objetivos específicos a cada categoria (VIEIRA et al., 2015).

De acordo com Françoso et al. (2015) 8,3\% da vegetação original do Cerrado é mantida sob proteção. Porém, é necessário compreender que a eficiência das UCs é dependente do tipo, do grau de restrição de uso, do envolvimento governamental e do tamanho de área protegida (PAIVA; BRITES; MACHADO, 2015). Dessa forma, o conhecimento das UCs é fundamental para o estabelecimento satisfatório de políticas públicas voltadas para a criação, o gerenciamento, a ampliação e a proteção das mesmas. Assim, o presente estudo tem como objetivo identificar a evolução das UCs ao longo de 70 anos no Cerrado, e compreender o atual estado de tais unidades no bioma.

\section{METODOLOGIA}

A área de estudo refere-se ao Cerrado brasileiro que se encontra predominantemente no planalto central do Brasil, nas latitudes $5^{\circ}$ a $20^{\circ} \mathrm{S}$ e longitude $45^{\circ}$ a $60^{\circ} \mathrm{O}$, com altitudes variando de quase 0 a 1800 m. A região está inserida em três importantes bacias hidrográficas (Tocantins-Araguaia, Paraná e São Francisco) refletindo em uma grande diversidade de solo e clima e, consequentemente, em uma fauna e flora diversificada. O bioma se distribui nos estados de Goiás, Tocantins e o Distrito Federal, parte dos estados da Bahia, Ceará, Maranhão, Mato Grosso, Mato Grosso do Sul, Minas Gerais, Piauí, Rondônia, São Paulo e em pequenas ilhas no Paraná (COUTINHO, 2016) (Figura 1).

Figura 1 - Cerrado: Localização geográfica, 2020.

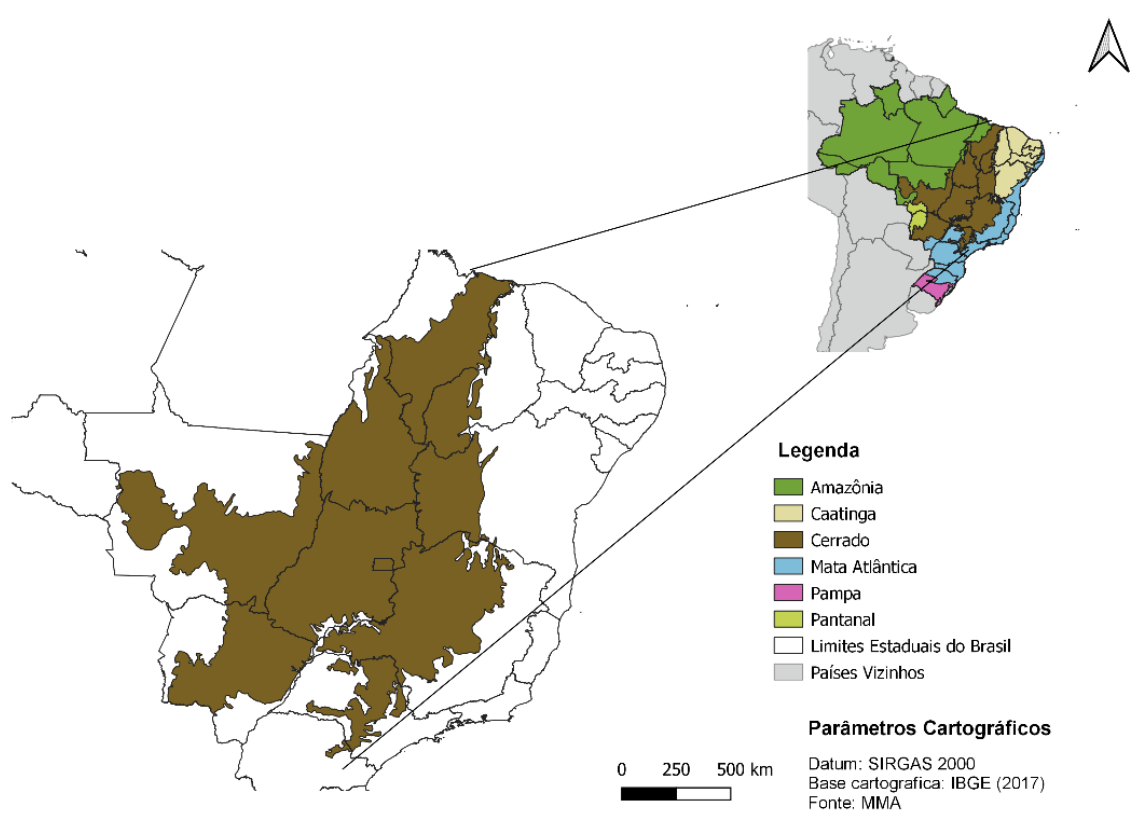

Fonte - AUTORES (2020). 
Para a realização desse estudo foram obtidos e tabulados dados públicos oficiais gerados pelo Ministério do Meio Ambiente (MMA), que se encontra na aba de Cadastro Nacional de Unidades de Conservação (MMA, 2020). As informações registradas referem-se ao ano de criação, tamanho (ha), grupo (Proteção Integral e Uso Sustentável) categorias (Estação Ecológica - EE; Reserva Biológica RB; Parque - PQ; Monumento Natural - MN; Refúgio de Vida Silvestre - RVS; Área de Proteção Ambiental - APA; Área de Relevante Interesse Ecológico - ARIE; Floresta - FL; Reserva Extrativista RE; Reserva de Fauna - RF; Reserva de Desenvolvimento Sustentável - RDS; Reserva Particular do Patrimônio Natural - RPPN), esfera administrativa (federal, estadual ou municipal) e a presença ou ausência do plano de manejo de cada Unidade de Conservação.

Para a confecção dos mapas, os limites das unidades federativas e os shapefiles das UCs foram obtidos através do Instituto Brasileiro de Geografia e Estatística (IBGE) na aba de bases cartográficas contínuas, na escala de 1:250000 (IBGE, 2017). Já os vetores dos biomas foram fornecidos pelo MMA através da plataforma i3Geo. Para a junção desses dados e elaboração do mapa de UCs no Cerrado foi utilizado o software QGIS 3.4.13.

\section{RESULTADOS E DISCUSSÃO}

A primeira Unidade de Conservação no Cerrado surgiu em 1949, por meio da lei no 612 que instituiu a criação do Horto Florestal de Silvânia (GO), sendo a mesma renomeada como Floresta Nacional em 2001 pela portaria no 247 (BRASIL, 1949, 2001). Na Figura 2, verifica-se a evolução na quantidade de UCs criadas por decênio nos últimos 70 anos. Esse processo não ocorreu de forma homogênea, apresentando um crescimento a partir do ano de 1970 com maior intensidade entre 1990 e 2009. De acordo com Watson et al. (2014), o reconhecimento da importância da conservação in situ levou a uma expansão de áreas protegidas no mundo na década de 70, quando os países se empenharam em conservar seus ecossistemas frente às rápidas mudanças da modernização global. Além disso, no ano 2000 foi estabelecido o SNUC, por meio da lei no 9.985 (Brasil, 2000), contribuindo para a expansão das UCs nessa época. Anteriormente a este período, duas novas leis foram publicadas e contribuíram com a expansão das UCs na década de 80 e 90, a lei no 6.938 (Brasil, 1981a), que dispõe sobre a Política Nacional de Meio Ambiente e a lei no 6.902 (Brasil, 1981b) que dispõe sobre a criação de Estações Ecológicas e Áreas de Proteção Ambiental.

Figura 2 - Cerrado: Evolução histórica de Unidades de Conservação, 2020.

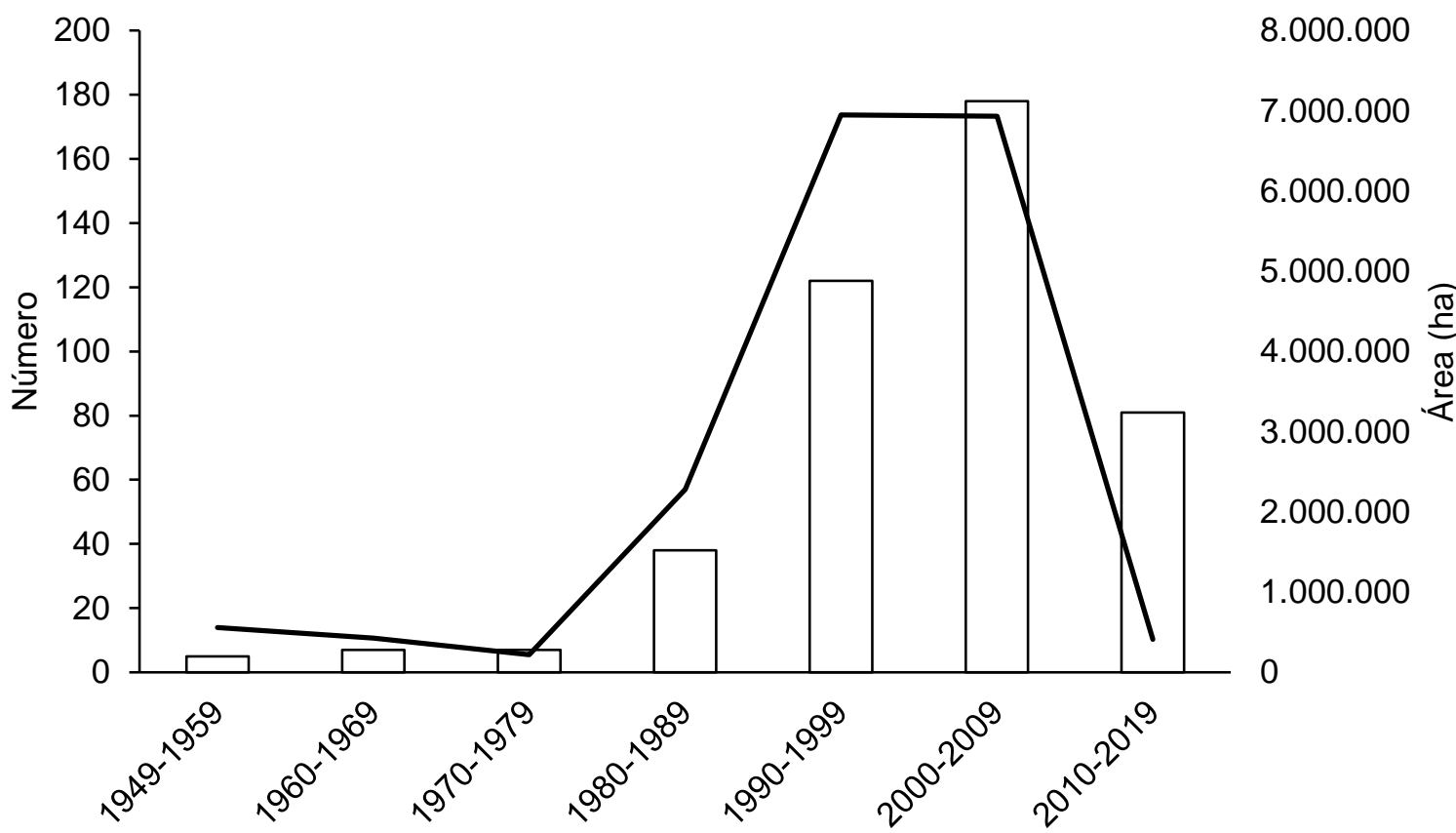

Periódo

Fonte - AUTORES (2020). 
Na última década, observa-se que houve uma acentuada queda na quantidade e na área total de UCs no Brasil. Esse fato relaciona-se a falta de investimentos de recursos financeiros e humanos para implantação, manutenção e gestão dessas áreas (BLACKMAN; PFAFF; ROBALINO, 2015). Além disso, a ausência de apoio governamental nas áreas de proteção contribui para esse cenário atual. Enquanto isso, a fronteira agrícola continua em rápida expansão dificultando a conversão de novas áreas para UCs.

O Cerrado brasileiro é o segundo maior bioma em área territorial (203.644.800 ha) (FERNANDES; FREDERICO; PEREIRA, 2019). Também ocupa a segunda posição em termos de quantidade e área de UCs (Tabela 1) que, conforme Ellison et al. (2017), desempenham um importante papel na proteção dos solos, sequestro de carbono, conservação das florestas nativas e regulação da temperatura e ciclo hidrológico. Além disso, podem contribuir com o desenvolvimento econômico das cidades (BARAL; GUARIGUATA; KEENAN, 2016). No entanto, de acordo com os dados deste trabalho, observa-se que apenas $8,72 \%$ da área do bioma encontra-se protegida. Em relação aos demais biomas, o Cerrado apresenta uma área protegida superior ao Pampa $(2,94 \%)$ e ao Pantanal $(4,63 \%)$. Porém, inferior a Caatinga $(8,80 \%)$, a Mata Atlântica $(9,84 \%)$ e a Amazônia (28\%). Este cenário demonstra a ineficiência das autoridades em realizar o compromisso estabelecido pelo Decreto oㅡ 5758/2006 (Brasil, 2006) de cumprir a meta de conservar 10\% do bioma Cerrado, definido pelo Programa de Trabalho para Áreas Protegidas da Convenção sobre Diversidade Biológica (MEDEIROS e ARAÚJO, 2011). Porém, isso se agrava quando comparado as metas de Aichi, definida durante a $10^{\text {a }}$ Conferência das Partes da Convenção sobre Diversidade Biológica no Japão, onde se estabeleceu como objetivo conservar $17 \%$ de áreas terrestres até 2020 (WATSON et al., 2014). Assim, visando cumprir os compromissos ambientais juntamente com o aumento das pressões antrópicas sob os ecossistemas nativos, é evidente a necessidade de definir cuidadosamente novas áreas para conservação.

Tabela 1 - Brasil: Número e área de Unidades de Conservação, 220.

\begin{tabular}{ccc}
\hline Bioma & Número & Área (ha) \\
\hline Amazônia & 351 & 120.277 .239 \\
Cerrado & 438 & 17.771 .936 \\
Mata Atlântica & 1364 & 11.750 .888 \\
Caatinga & 201 & 7.502 .915 \\
Pampa & 33 & 569.650 \\
Pantanal & 25 & 695.004 \\
\hline Total & $\mathbf{2 4 1 2}$ & $\mathbf{1 5 8 . 5 6 7 . 6 3 3}$
\end{tabular}

Fonte - AUTORES (2020).

Atualmente o Cerrado possui cerca de 438 UCs. Dessas, 32\% (142 UCs) pertencem ao grupo de PI, que ocupam uma área de 6.310 .110 ha (3,1\% da área do Cerrado); e 68\% (296) estão presentes no grupo de US 11.461 .826 ha $(5,62 \%$ da área do Cerrado) (Tabela 2). Em ordem decrescente, a ocupação em ha de cada categoria em relação a extensão territorial do Cerrado é: APA $(5,45 \%), P Q$ $(2,5 \%)$, EE $(0,45 \%)$, RVS $(0,12 \%)$, RE $(0,06 \%)$, RPPN $(0,06 \%)$, RDS $(0,03 \%)$, FL $(0,03 \%)$, MN $(0,02 \%)$, RB $(0,01 \%)$ e ARIE $(0,01 \%)$. Como visto, grande parte das unidades são classificadas como APA, categoria menos restritiva em relação ao uso permitido dos recursos naturais. Além disso, ressalta-se que até 20 julho de 2020, não ocorreu registro de Reservas de Fauna para o Cerrado no Cadastro Nacional de Unidades de Conservação. No entanto, essas seriam fundamentais para apoiar os trabalhos técnico-científicos que permitiriam o aproveitamento econômico e o manejo sustentável dos recursos obtidos pela fauna.

De acordo com Peres (2011), no Brasil existe uma forte tendência para a criação de unidades de US. No entanto, essas áreas apresentam uma pequena contribuição a conservação da biodiversidade, pois permitem o uso do solo para atividades agrícola e pecuária, além da permanência humana em seu interior (MARQUES; SCHNEIDER; PERES, 2016). Os mesmos autores relataram que a ocupação antrópica nas UCs aumenta as taxas de desmatamento e incêndio, promovendo a perda de habitat. Françoso et al. (2015) também observaram que as áreas de US apresentaram maiores níveis de desmatamento, em comparação com as unidades de PI. Todo esse processo revela a ineficiência de

$\begin{array}{lllll}\text { Caminhos de Geografia } & \text { Uberlândia-MG } & \text { v. 22, n. } 83 & \text { out./2021 } & \text { p. 133-143 Página } 136\end{array}$


muitas unidades de US para a conservação da natureza, que se agrava com as falhas na gestão e na fiscalização dessas áreas, sendo aconselhável adotar um maior incentivo para a criação de áreas de $\mathrm{PI}$, visto que essas garantem uma maior proteção dos ecossistemas.

Tabela 2 - Cerrado: Número e área por categoria de Unidades de Conservação, 2020.

\begin{tabular}{ccc}
\hline \multicolumn{3}{c}{ Proteção Integral } \\
\hline Categoria & Quantidade & Área (ha) \\
\hline EE & 26 & 918.495 \\
RB & 7 & 12.038 \\
PN & 88 & 5.095 .663 \\
MN & 15 & 37.642 \\
RVS & 6 & 246.272 \\
\hline Total & $\mathbf{1 4 2}$ & $\mathbf{6 3 1 0 1 1 0}$ \\
\hline UPA & Usostentável & 11.092 .975 \\
ARIE & 86 & 11.320 \\
FN & 20 & 55.686 \\
RE & 11 & 115.188 \\
RDS & 7 & 68.660 \\
RPPN & 2 & 117.997 \\
\hline Total & 170 & $\mathbf{1 1 4 6 1 8 2 6}$ \\
\hline
\end{tabular}

Fonte - AUTORES (2020).

Aproximadamente $40 \%$ de UCs são classificadas como RPPN. Embora em maior número de UCs, a RPPN é uma categoria composta por unidades de pequena extensão. Essas reservas geralmente são incorporadas em uma paisagem altamente fragmentada, resultando na limitação do fornecimento dos serviços ecossistêmicos (MELLO; TOPPA; CARDOSO-LEITE, 2016). Porém, podem contribuir com a conectividade entre fragmentos maiores e funcionar como refúgio para fauna caso ocorram distúrbios naturais ou antrópicos em outras áreas (MARQUES; SCHNEIDER; PERES, 2016). Além disso, exigem pouco investimento financeiro do governo, visto que são de propriedade e administração privada.

As UCs do Cerrado variam muito em tamanho, apresentando áreas que variam de 1 a aproximadamente 1,5 milhões de ha. As maiores unidades de conservação de US são a Área de Proteção Ambiental do Rio Preto (1.107.499 ha) e a Área de Proteção Ambiental llha do Bananal/Cantão (1.570.008 ha). As maiores de PI são o Parque Nacional das Nascentes do Rio Parnaíba (749.766 ha) e o Parque Estadual de Mirador (766.781 ha). No entanto, observa-se que, aproximadamente, $90 \%$ das UCs apresentam área menor que 100.000 ha, desse total $53,4 \%$ possuem área entre 1 e 1.000 ha (Figura 3). Essas áreas em menor extensão são um grande desafio ambiental, pois, geralmente, estão inseridas dentro de regiões densamente povoadas, recebendo ameaças antrópicas e, consequentemente, não garantindo a conservação da biodiversidade local (SALVIO; GOMES, 2018). Alguns autores como Soulé e Wilcox (1980) e Primack e Rodrigues (2001) relatam que para uma unidade de conservação manter seus processos e funções ecológicas ativos, devem possuir no mínimo 100.000 ha. Além disso, um dos aspectos que devem ser considerados pelo governo para definir as áreas destinadas a conservação, restauração e uso sustentável é a conectividade que ela pode fornecer entre os fragmentos de vegetação nativa presente na paisagem (SANO et al., 2010). Assim, é fundamental estabelecer conexões entre as UCs, através da implementação de corredores ecológicos, pois o isolamento dessas unidades tende a levar a extinção das populações vegetais (LANDE, 1998; LEVERINGTON et al., 2010).

Caminhos de Geografia Uberlândia-MG

v. 22, n. 83

out./2021

p. $133-143$

Página 137 
Figura 3 - Cerrado: Número por tamanho de área de Unidades de Conservação, 2020.

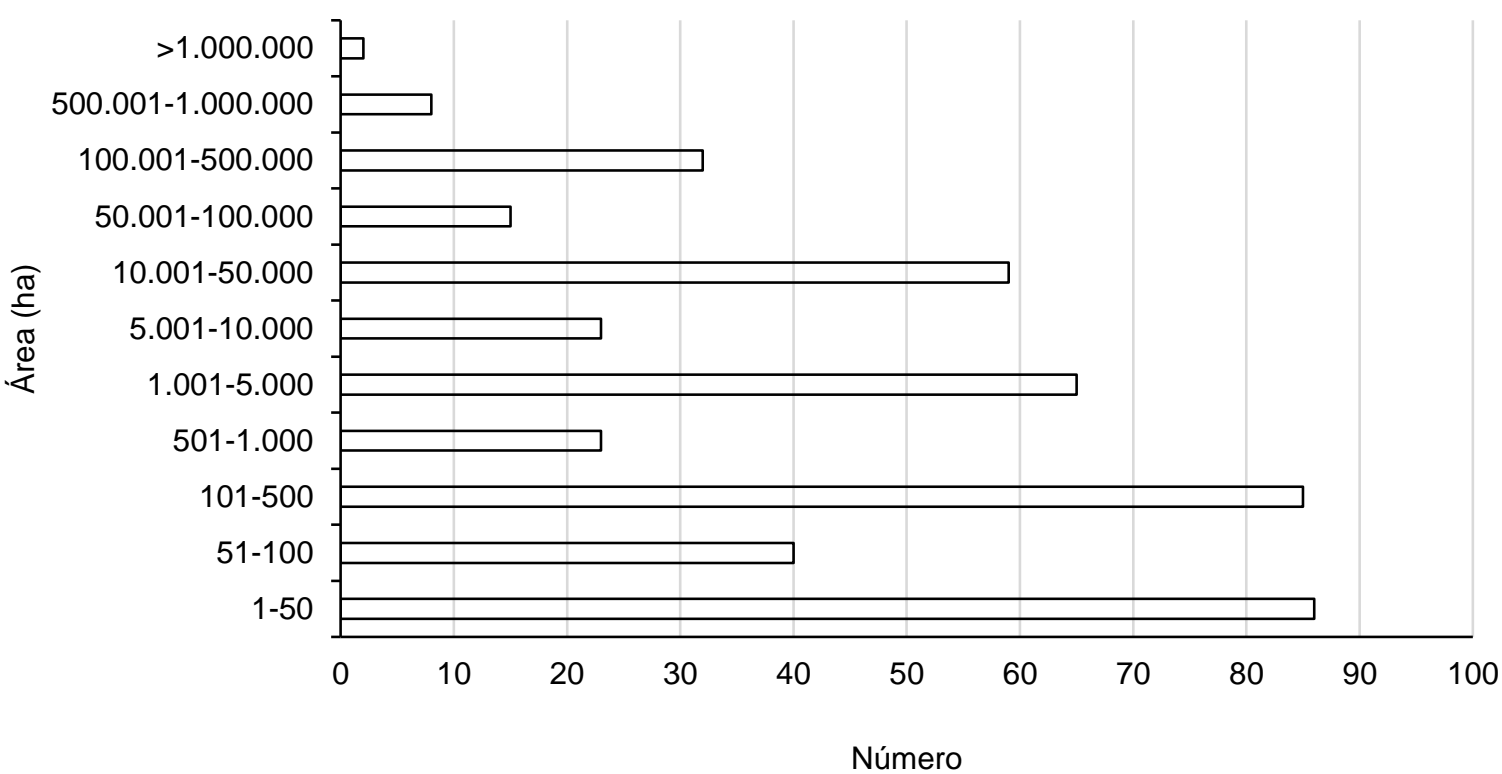

Fonte - AUTORES (2020).

Outro aspecto a ser planejado no processo de criação e gestão das UCs é a ocupação do solo no entorno das unidades. Se as UCs estão inseridas em uma região degradada por ações antrópicas tornam-se fragmentos isolados na paisagem, afetando a estrutura, o equilíbrio, a saúde, a biodiversidade, os processos ecológicos e a resiliência desses ecossistemas (ANDREASEN et al., 2001). Dessa forma, é importante a criação de Zonas de Amortecimento, pois essas funcionarão como filtros aos impactos negativos das atividades que ocorrem no exterior da unidade. Além disso, é necessária uma maior parceria entre os órgãos governamentais e os grupos ambientais, juntamente com os proprietários e trabalhadores rurais do entorno das UCs, pois a permanência da cobertura vegetal nativa depende em grande parte deles. Por exemplo, apesar do número significativo de UCs no Distrito Federal, a biodiversidade externa das unidades está comprometida, resultando em diversas UCs degradadas com um estado frágil, diante das pressões geradas pelas populações vizinhas (GANEM; DRUMMOND; FRANCO, 2013).

As UCs estão inseridas em diferentes esferas administrativas, federal, estadual e municipal, sob gestão do ICMBio, órgãos ambientais estaduais e órgãos ambientais municipais, respectivamente. Do total de UCs, 177 federais, 96 estaduais e 32 municipais são do grupo de US. Em contrapartida, 20 federais, 98 estaduais e 15 municipais são de PI. No Cerrado, observa-se que as UCs federais se sobressaem às estaduais, e as municipais apresentam menor número (Tabela 3). No entanto, a quantidade de UCs sob regime estadual é significativa, o que pode ser explicado pela presença do Sistema Estadual de Unidades de Conservação (SEUC) em quase todos os estados que o bioma Cerrado ocupa. O estado do Piauí é desprovido de tal legislação, o que justificar a ausência de UCs estaduais na região. Já os estados de Minas Gerais e Mato Grosso do Sul, apesar de apresentarem UCs sob sua administração, não possuem SEUC (SALVIO et al., 2020).

Ao analisar as UCs sem plano de manejo por esfera administrativa, constata-se que o menor índice é obtido em nível municipal (10,86\%), seguido do estadual $(39,68 \%)$ e federal $(49,46 \%)$. Os dados também demostraram que $93(25,27 \%)$ e $275(74,73 \%)$ UCs de PI e US, respectivamente, não possuem plano de manejo, sendo este um documento essencial para a gestão física e dos recursos naturais de áreas protegidas. De acordo com Lima; Ribeiro; Gonçalves (2005) os dirigentes públicos preocupamse em criar UCs sem planejar a sua implantação e gestão, criando apenas unidades no papel, porém, sem funcionários, infraestrutura, plano de proteção, controle de incêndios e plano de manejo. Assim, infelizmente, os interesses políticos e econômicos são geralmente os critérios mais importantes no momento da escolha do local de uma UCs, e não os científicos (DINIZ-FILHO et al., 2004).

A lei no 9.985 estabelece que o plano de manejo deve ser elaborado no prazo de cinco anos a partir da data de criação de uma unidade de conservação (BRASIL, 2000). Existem diversas UCs criadas em

$\begin{array}{lllll}\text { Caminhos de Geografia } & \text { Uberlândia-MG } & \text { v. 22, n. } 83 & \text { out./2021 } & \text { p. 133-143 Página } 138\end{array}$


período superior a esse determinado pelo SNUC e que até 20 julho de 2020 não apresentavam plano de manejo, tais como: Monumento Natural da Gruta do Lago Azul (ano de criação: 2001, município: Bonito - MS), Área de Proteção Ambiental das Bacias do Rio Aporé e do Rio Sucuriu (2005, Chapadão do Sul - MS), Reserva Particular do Patrimônio Natural Arara Vermelha (2005, Arinos - MG), Área de Proteção Ambiental de São Desidério (2006, São Desidério - BA), Parque Estadual Vale do Códo (2007, Jaguariaíva - PR), Reserva Particular do Patrimônio Natural Buraco das Araras (2007, Jardim - MS), Área de Proteção Ambiental Jupiá (2009, Três Lagoas - MS), Monumento Natural Estadual Várzea da Lapa (2010, Lagoa Santa - MG), Parque Natural Municipal do Tabuleiro (2013), etc.

Tabela 3 - Cerrado: Unidades de Conservação com presença ou ausência de plano de manejo por esfera administrativa, 2020.

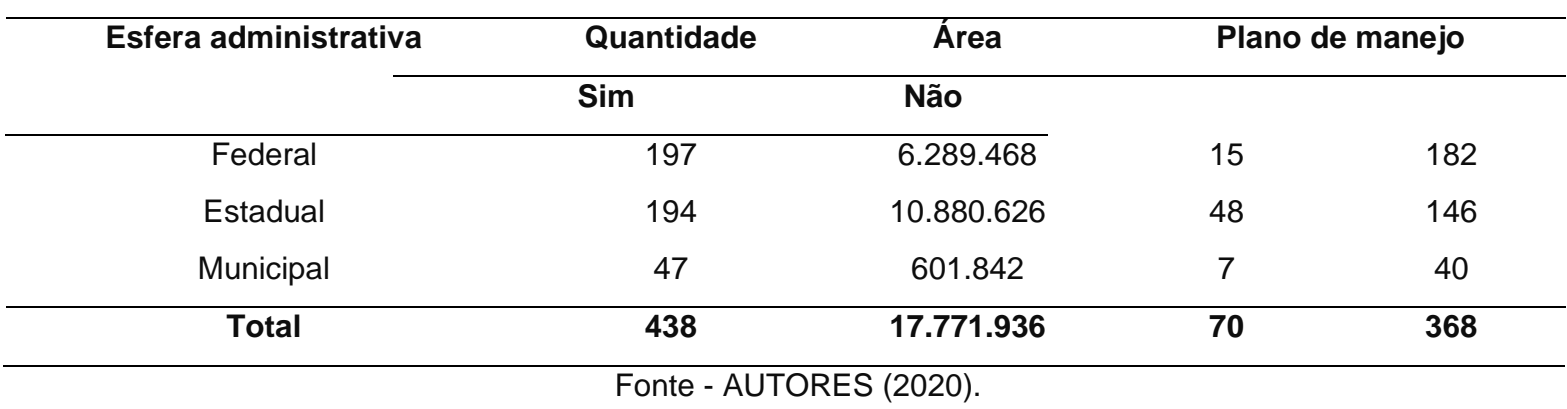

A Figura 4 exemplifica o número de UCs por unidade federativa no Cerrado. Os estados do Maranhão, Bahia e Paraná registram uma baixa quantidade de UCs. No entanto, embora os estados de Minas Gerais e Goiás possuam a maior quantidade de UCs, grande parte dessas são classificadas como RPPN: 70 (32.782 ha) e 60 (35.748 ha) unidades, respectivamente.

Figura 4 - Cerrado: Número de Unidades de Conservação por unidade federativa, 2020.

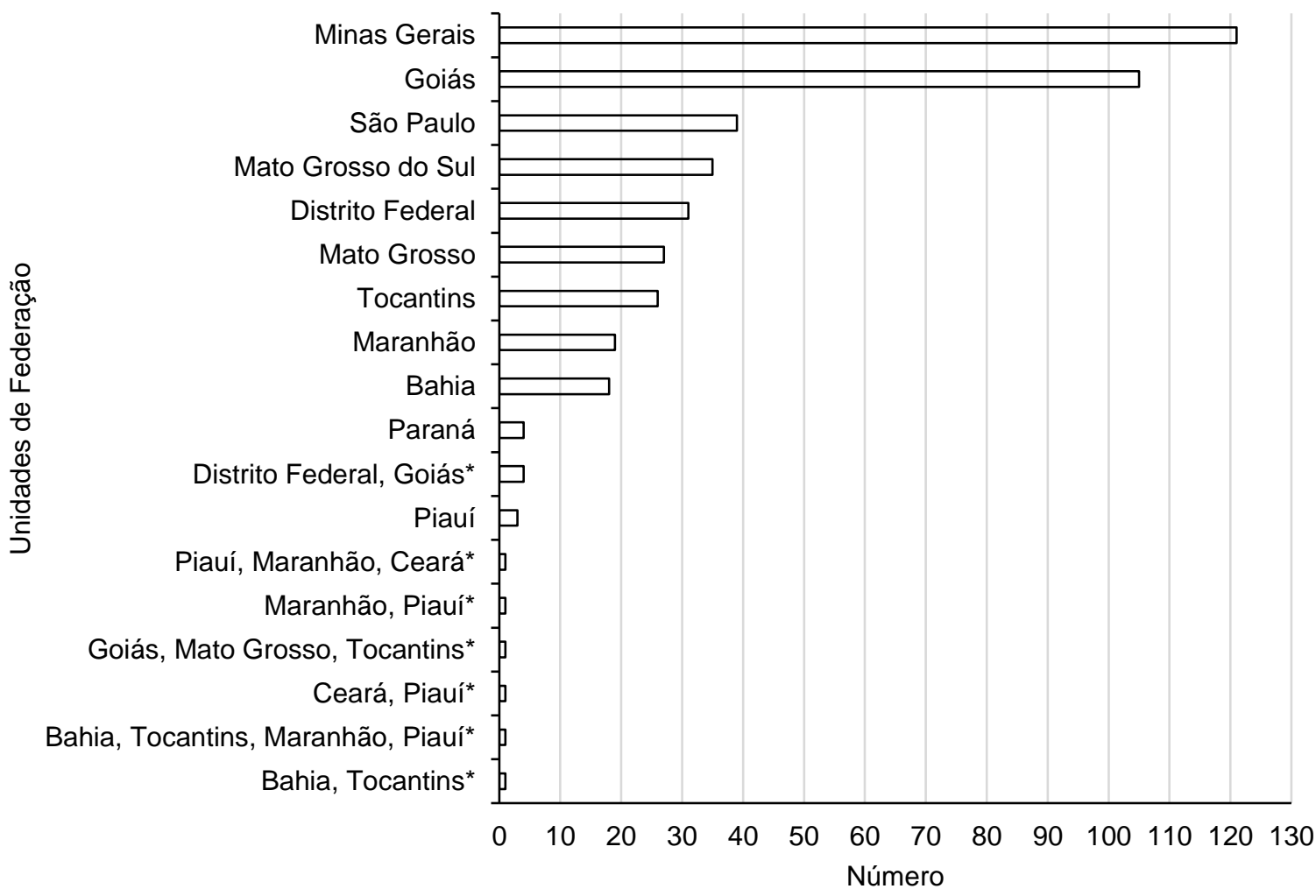

Fonte - AUTORES (2020)

$\begin{array}{lllll}\text { Caminhos de Geografia } \quad \text { Uberlândia-MG } & \text { v. 22, n. } 83 & \text { out./2021 } & \text { p. 133-143 } & \text { Página } 139\end{array}$




\section{CONSIDERAÇÕES FINAIS}

Uma pequena porcentagem do Cerrado se encontra protegida através das Unidades de Conservação, longe de cumprirem as metas de conservação. As unidades de Proteção Integral apresentam menor área e número em comparação com as de Uso Sustentável, sendo que essas ultimas realizam uma menor proteção da biodiversidade dos ecossistemas. Assim, a proteção do bioma Cerrado é ineficiente perto da importância do mesmo para a conservação da biodiversidade e do patrimônio genético brasileiro, sendo de grande relevância o aumento dessas áreas, principalmente, o grupo de Proteção Integral.

É necessária uma maior urgência na elaboração de estratégias governamentais a fim de criar novas UCs. No entanto, essas devem apresentar tamanho adequado e estarem integradas as demais UCs na paisagem. Além disso, é essencial que o entorno das atuais e futuras UCs sejam protegidos. Também é fundamental uma maior atenção dos órgãos ambientais para o cumprimento da legislação em relação a elaboração do plano de manejo, pois a ausência desse documento deixa as UCs vulneráveis aos conflitos ambientais e sem orientação clara para atuação dos fiscais.

Por fim, acreditamos que as informações contidas neste estudo podem auxiliar os órgãos públicos na orientação de diretrizes de planejamento da ocupação espacial das áreas protegidas e do seu entorno, representando um passo importante na busca pelo aperfeiçoamento da gestão e manutenção das Unidades de Conservação no Cerrado brasileiro.

\section{REFERÊNCIAS}

ANDREASEN, J. K. et al. Considerations for the development of a terrestrial index of ecological integrity. Ecological Indicators, v. 1, p. 21-35, 2001. https://doi.org/10.1016/S1470-160X(01)00007-3

ARRUDA, F. V. et al. Trends and gaps of the scientific literature about the effects of fire on Brazilian Cerrado. Biota Neotropica, v. 18, p. 10-26, 2018. https://doi.org/10.1590/1676-0611-bn-2017-0426

AZEVEDO-SANTOS, V. M. et al. Protected areas: A focus on Brazilian freshwater biodiversity. Diversity and Distributions, v. 25, p. 442-448, 2018. https://doi.org/10.1111/ddi.12871

BARAL, H.; GUARIGUATA, M. R.; KEENAN, R. J. A proposed framework for assessing ecosystem goods and services from planted forests. Ecosystem Services, v. 22, p. 260-268, 2016. https://doi.org/10.1016/i.ecoser.2016.10.002

BLACKMAN, A.; PFAFF, A.; ROBALINO, J. Paper park performance: Mexico's protected natural areas in the 1990s. Global Environmental Change, v. 31, p. 50-61, 2015.

https://doi.org/10.1016/j.gloenvcha.2014.12.004

BRASIL. Lei no 612, de 13 de janeiro de 1949. Disponível em:

https://www2.camara.leg.br/legin/fed/lei/1940-1949/lei-612-13-janeiro-1949-347995-

publicacaooriginal-1-pl.html Acesso em: 11 de janeiro de 2020.

BRASIL. Lei no 6.938, de 31 de agosto de 1981a. Disponível em:

http://www.planalto.gov.br/ccivil_03/LEIS/L6938.htm\#: :text=LEl\%20N\%C2\%BA\%206.938\%2C\%20D E\%2031\%20DE\%20AGOSTO\%20DE\%201981\&text=Disp\%C3\%B5e\%20sobre\%20a\%20Pol\%C3\%A Dtica\%20Nacional,Lei\%2C\%20com\%20fundamento\%20no\%20art. Acesso em: 04 de janeiro de 2020.

BRASIL. Lei no 6.902, de 27 de abril de 1981b. Disponível em:

http://www.planalto.gov.br/ccivil_03/LEIS/L6902.htm\#: :text=LEI\%20No\%206.902\%2C\%20DE\%2027 \%20DE\%20ABRIL\%20DE\%201981.\&text=Disp\%C3\%B5e\%20sobre\%20a\%20cria\%C3\%A7\%C3\%A3 \%\%20de,Art\%20. Acesso em: 04 de janeiro de 2020.

BRASIL. Lei no 9.985, de 18 de julho de 2000. Disponível em: http://www.planalto.gov.br/ccivil_03/leis/19985.htm Acesso em: 11 de janeiro de 2020.

BRASIL. Portaria $\mathbf{n}^{\circ}$ 247, de 18 de julho de 2001. Disponível em: https://www2.camara.leg.br/legin/marg/portar/2001/portaria-247-18-julho-2001-387481publicacaooriginal-1-mma.html Acesso em: 25 de janeiro de 2020. 
BRASIL. Decreto no 5.758, de 13 de abril de 2006. Disponível em: http://www.planalto.gov.br/ccivil_03/_Ato2004-2006/2006/Decreto/D5758.htm Acesso em: 24 de março de 2020.

COUTINHO, L. M. Biomas brasileiros. São Paulo: Oficina de Textos, 2016.

DINIZ-FILHO, J. A. F. et al. Spatial patterns in species richness and priority áreas for conservation of anurans in the Cerrado region, Central Brazil. Amphibia-Reptilia, v. 25, p. 63-75, 2004. https://doi.org/10.1163/156853804322992841

ELLISON, D. et al. Trees, forests and water: Cool insights for a hot world. Global Environmental Change, v. 43, p. 51-61, 2017. https://doi.org/10.1016/i.gloenvcha.2017.01.002

FEARNSIDE, P. M. Deforestation in Brazilian Amazonia: history, rates, and consequences. Conservation Biology, v. 19, p. 680-688, 2005. https://doi.org/10.1111/i.1523-1739.2005.00697.x

FELFILI, J. M.; SILVA JUNIOR, M. C. A comparative study of Cerrado (sensu stricto) vegetation in Central Brazil. Acta Botanica Brasilica, v. 9, p. 277-289, 1993. https://doi.org/10.1017/S0266467400007306

FERNANDES, B. M.; FREDERICO, S.; PEREIRA, L. I. Acumulação pela renda da terra e disputas territoriais na fronteira agrícola brasileira. Revista Nera, v. 22, p. 173-201, 2019.

FRANÇOSO, R. D. et al. Habitat loss and the effectiveness of protected areas in the Cerrado Biodiversity Hotspot. Natureza e conservação, v. 13, p. 35-40, 2015.

https://doi.org/10.1016/i.ncon.2015.04.001

GANEM, R. S.; DRUMMOND, J. A.; FRANCO, J. L. A. Conservation policies and control of habitat fragmentation in the brazilian Cerrado bioma. Ambiente \& Sociedade (Online), v. 16, p. 99-118, 2013. https://doi.org/10.1590/S1414-753X2013000300007

GIBBS, H. K. et al. Brazil's Soy Moratorium. Science, v. 347, p. 377-378, 2015.

https://doi.org/10.1126/science.aaa0181

IBGE. Instituto Brasileiro de Geografia e Estatística. Disponível em:

https://portaldemapas.ibge.gov.br/portal.php\#homepage Acesso em: 14 de março de 2020.

LANDE, R. Extinction risks from antropogenia, ecological and genetic factors. In: LANDWEBER, L. F.; DOBSON. A. P. (Eds.). Genetics and the extinction of species. Princeton: Princeton University Press, 1998. p. 50-96.

LEVERINGTON, F. et al. A global analysis of protected area management effectiveness. Environmental Management, v. 46, p. 685-698, 2010. https://doi.org/10.1007/s00267-010-9564-5

LIMA G. S.; RIBEIRO, G. A.; GONÇALVES, W. Avaliação da efetividade de manejo das unidades de conservação de proteção integral em Minas Gerais. Revista Arvore, v. 29, p. 647-653. 2005. https://doi.org/10.1590/S0100-67622005000400017

MARQUES, A. A. B.; PERES, C. A. Pervasive legal threats to protected areas in Brazil. Fauna e Flora International, v. 49, p. 25-29, 2015. https://doi.org/10.1017/S0030605314000726

MARQUES, A. A. B.; SCHNEIDER, M.; PERES, C. A. Human population and socioeconomic modulators of conservation performance in 788 Amazonian and Atlantic Forest reserves. PEERJ, v. 4, p. 1-27, 2016. https://doi.org/10.7717/peeri.2206

MAPBIOMAS. Relatório Anual do Desmatamento no Brasil 2019. Disponível em: https://s3.amazonaws.com/alerta.mapbiomas.org/relatrios/MBI-relatorio-desmatamento-2019FINAL5.pdf Acesso em: 21 de dezembro. 2020.

MEDEIROS, R.; ARAÚJO, F. S. A. Dez anos do Sistema Nacional de Unidades de Conservação da Natureza: lições do passado, realizações presentes e perspectivas para o futuro. Brasília: MMA, 2001.

MELLO, K. de.; TOPPA, R. H.; CARDOSO-LEITE, E. Priority areas for forest conservation in an urban landscape at the transition between atlantic forest and Cerrado. Cerne, v. 22, p. 277-288, 2016. https://doi.org/10.1590/01047760201622032172

MMA. Ministério do Meio Ambiente. Disponível em: https://www.mma.gov.br/areasprotegidas/cadastro-nacional-de-ucs Acesso em: 20 de julho de 2020.

$\begin{array}{lllll}\text { Caminhos de Geografia } & \text { Uberlândia-MG } & \text { v. 22, n. } 83 & \text { out./2021 } & \text { p. 133-143 Página } 142\end{array}$


OLIVEIRA, H. R. et al. Geographical ecology and conservation of Eugenia L. (Myrtaceae) in the Brazilian Cerrado: past, present and future. Austral Ecology, v. 43, p. 3-10, 2018.

PAIVA, R. J. O.; BRITES, R. S.; MACHADO, R. B. The Role of Protected Areas in the Avoidance of Anthropogenic Conversion in a High Pressure Region: A Matching Method Analysis in the Core Region of the Brazilian Cerrado. PLoS One, v. 10, p. 1-24, 2015.

https://doi.org/10.1371/journal.pone.0132582

PERES, C. A. Conservation in sustainable-use tropical forest reserves. Conservation Biology, v. 25, p. 1124-1129, 2011. https://doi.org/10.1111/j.1523-1739.2011.01770.x

PRIMACK, R. B.; RODRIGUES, E. Biologia da Conservação. Londrina: Planta, 2001.

SALVIO, G. M. M.; et.al. Sistemas estaduais de unidades de conservação do Brasil. Revista Tecnológica e Sociedade, v. 16, p. 113-131, 2020. https://doi.org/10.3895/rts.v16n39.8978

SALVIO, G. M. M.; GOMES, C. R. Protected Area Systems in South American Countries. Floresta Ambiente, v. 25, p. 1-11, 2018. https://doi.org/10.1590/2179-8087.113417

SANO, E. E. et al. Land use dynamics in the Brazilian Cerrado in the period from 2002 to 2013.

Pesquisa Agropecuaria Brasileira, v. 54, p. 1-5, 2019.

SOULÉ, M. E.; WILCOX, B. A. Conservation Biology: an evolutionary-ecological perspective. Massachusetts: Sinauer, 1980.

STRASSBURG, B. B. et al. Moment of truth for the Cerrado hotspot. Nature Ecology \& Evolution, v. 1, p. 1-7, 2017. https://doi.org/10.1038/s41559-017-0099

VIEIRA, C. K. et al. Microbiome of a revegetated iron-mining site and pristine ecosystems from the Brazilian Cerrado. Applied Soil Ecology, v. 131, p. 55-65, 2018.

https://doi.org/10.1016/j.apsoil.2018.07.011

VIEIRA, R. M. S. P. et al. Identifying areas susceptible to desertification in the Brazilian northeast.

Solid Earth, v. 6, p. 347-360, 2015. https://doi.org/10.5194/se-6-347-2015

WATSON, J. E. M. et al. The performance and potential of protected áreas. Nature, v. 515, p. 67-73, 2014. https://doi.org/10.1038/nature13947

Recebido em: 21/07/2020

Aceito para publicação em: 05/12/2020 